\title{
Consideraciones ético-politico-afectivas en investigaciones feministas: articulaciones situadas entre academia y activismo
}

Ethical-political-affective considerations in feminist research: located articulations between academia and activism

\author{
Daniela Osorio-Cabrera \\ Universidad de la República- Uruguay \\ dosorio@psico.edu.uy (URUGUAY) \\ ItZIAR GANDARIAS \\ Universidad de Deusto \\ itziar.gandarias@deusto.es (ESPAÑA) \\ Karina Fulladosa \\ Universidad de la República- Uruguay \\ karinafulladosa@gmail.com (URUGUAY)
}

Recibido: 01.07.2019

Aceptado: 03.03.2021

\section{RESUMEN}

Este artículo tiene como objetivo realizar reflexiones teórico-epistemológicas en base a nuestras experiencias articuladas de investigación activista con perspectiva feminista. En base a un proceso de reflexión dialógica conjunta, compartimos algunas pistas de las dimensiones ético-político-afectivas de los procesos de investigación. Para ello ponemos en diálogo nuestros procesos de investigación con las epistemologías feministas y el intercambio con otras pensadorasinvestigadoras feministas.

Proponemos poner en valor las relaciones de afecto y amistad, que permiten que nuestras investigaciones sean más habitables. Apostamos por una reflexividad que se deja afectar y atravesar por las emociones como herramienta del conocimiento. Incorporamos el análisis semiótico-material de nuestros propios cuerpos, no para caer en la autorreferencia, sino para contemplar sus efectos en 
la producción de conocimiento. En síntesis, proponemos otras posibilidades de producción de conocimiento desde las experiencias corporales, los afectos y las diversas relaciones que vamos tejiendo y que sostienen nuestros procesos de investigación para construir colectivamente un conocimiento encarnado y situado, comprometido con la constitución de modos de vida vivibles.

\title{
PALABRAS CLAVE
}

Investigación Activista-feminista, Conocimientos Situados, Etico-politica, afectos.

\begin{abstract}
The aim of this article is to make theoretical-epistemological reflections based on our articulated experiences of activist research with a feminist perspective. Based on a process of joint dialogical reflection, we share some clues about the ethical-political-affective dimensions of the research processes. To this end, we put our research processes in dialogue with feminist epistemologies and the exchange with other feminist thinkers-researchers.

We propose to value affective relationships and friendship, which allow our research to be more habitable. We are committed to a reflexivity that allows itself to be affected and traversed by emotions as a tool for knowledge. We incorporate the semiotic-material analysis of our own bodies, not to fall into self-reference, but to contemplate their effects on the production of knowledge. In short, we propose other possibilities for the production of knowledge based on bodily experiences, affects and the diverse relationships that we weave and that sustain our research processes in order to collectively build an embodied and situated knowledge, committed to the constitution of livable ways of life.
\end{abstract}

\section{KEY WORDS}

Activist-feminist research, situated knowledge, ethics-politics, affection.

\section{LA ESPIRAL COMO METÁFORA DE LAS FORMAS DE PRODUCIR CONOCIMIENTO FEMINISTA.}

Las reflexiones que compartimos en este artículo tienen un recorrido en espiral. Nos gusta esta metáfora porque nos propone movernos en otro espaciotiempo, evitando una lectura lineal de nuestros procesos de investigación. Una espiral que articula ideas y reflexiones a partir del intercambio y las conversaciones que hemos construido en el "entre academia-activismo". Espiral que 
como señala Karina Fulladosa-Leal (2017) nos hace transitar por nuestras investigaciones a veces pasando por los mismos lugares en apariencia. Sin embargo, nuestra posición se transforma en este proceso, y esto se convierte en un acto de reflexión y conocimiento.

La espiral tiene uno de sus puntos de encuentro en nuestros trabajos de investigación en el Máster y luego en el Doctorado en Psicología Social de la Universidad Autónoma de Barcelona ${ }^{1}$. Nos referiremos a las reflexiones que comienzan a articularse en el devenir activista en tres experiencias de investigación con colectivas: en Sindihogar- Sindillar ${ }^{2}$ (Sindicato de trabajadoras del hogar y cuidado de Cataluña), estudiando la acción colectiva y el trabajo de cuidados y migraciones; en el Ateneo Cooperativo La Base en Barcelona ${ }^{3}$, estableciendo el diálogo entre economía solidaria y la economía feminista; y en la Plataforma de la Marcha Mundial de Mujeres en Euskal Herria ${ }^{4}$ analizando la articulación entre mujeres migradas y feministas autónomas vascas. El tránsito por nuestros espacios de formación e investigación, se convirtió entonces en un entretejido basado en la amistad y las complicidades teórico-políticas, un espacio de reflexiónacción conjunta que con el tiempo llamamos íntimamente "las marietas".

En este recorrido los feminismos nos atravesaron, reconociendo en particular los aportes desde las epistemologías feministas. Nos sentimos parte del entramado de reflexiones que las distintas vertientes dentro de los feminismos han aportado a la producción de conocimiento (Adan 2006). Partimos de la crítica de un conocimiento científico en el que predomina una mirada androcéntrica, no solo de las concepciones sobre las mujeres, sino también en la estructura institucional que la conforma y las finalidades que establece (Blázquez 2011). Nos referimos tanto a los cuestionamientos en torno a los sesgos de género que señala el empirismo feminista, a la mirada desde los márgenes que nos plantea el punto de vista feminista (Harding 1996; Keller 1991), así como al reconocimiento de nuestras posiciones a la hora de producir conocimiento (Haraway 1997). Por último, compartimos el compromiso de poner en práctica otras formas de (re) conocer, más horizontales, ética y políticamente responsables (Adán 2006) con mayor orientación hacia la transformación social.

En particular nuestros trabajos fueron inspirados por los planteos del conocimiento situado de Donna Haraway (1997). Nos interesa destacar de su propuesta la necesidad de posicionarnos en los procesos de investigación, para construir objetividad desde la articulación de las distintas posiciones. Por eso nos parece importante hablar de la construcción de un conocimiento sucio (Haraway 1997), que nos encuentra embarradas en nuestros procesos de investigación. Cuando decimos embarradas, hacemos alusión al involucramiento en las investigaciones, a poner el cuerpo siendo parte de un proceso que nos compromete a decir cosas

\footnotetext{
${ }^{1}$ Las tres coincidimos realizando el Máster de investigación en Psicología Social durante el curso 2011/2012 y después durante los años de Doctorado.

2 https://sindillar.org/

3 http://labase.info

4 http://www.emakumeenmundumartxa.eus
} 
del mundo. Sucio también, por alejarse de la lógica de la representación pura y el conocimiento neutro. Buscamos hacer visible nuestra intervención en los procesos de investigación, con base en el reconocimiento del encuentro de las distintas posiciones que habitamos las investigadoras y las personas o colectivos con los que nos involucramos; encuentro que no romantizamos pues precisamente está lleno de conflictos, diferencias y tensiones.

Sin embargo, es un conocimiento transparente, precisamente, porque hace visible eso que llamamos la cocina de la investigación. El relato sobre las dudas, los aciertos y errores del camino, las intuiciones, los malestares, todo aquello que forma parte de nuestros procesos de investigación pero que pocas veces son nombrados en los textos académicos. Esta transparencia es la que permite también una forma de validar nuestro conocimiento, al estar abiertas a un análisis crítico de los mismos. Dicho en las palabras de Haraway (1997:29):

“... el objetivo es marcar una diferencia en el mundo, implicarse por unos modos de vida y no otros. Para hacerlo, hay que estar en la acción, ser finito y sucio, no trascendente y limpio. Las tecnologías que producen conocimiento, incluyendo la creación de posiciones de sujeto y los modos de habitar tales posiciones, deben hacerse visibles sin vacilar y estar abiertas a la intervención crítica".

Nos posicionamos dentro de los colectivos marcando ese espacio fronterizo "entre" la dimensión material y afectiva, "entre" la teoría y la práctica, "entre" la acción y la reflexión, "entre" unas y otras. Esta estrategia se inspira en los planteos de Bárbara Biglia (2005), en relación a la propuesta de una Investigación Activista Feminista (IAF).

Es decir, una propuesta de investigación comprometida con el cambio social, que nos convoca a pensarnos como acompañantes de un proceso de construcción de saberes colectivos. La IAF se basa en una búsqueda constante por romper la dicotomía público-privado, en la que nos implicamos desde una visión crítica. Una crítica no solo de lo social sino de lo personal como investigadoras, cuestionando siguiendo a Blázquez (2008) nuestros propios esquemas culturales androcéntricos.

La IAF es una propuesta que reconoce, valora y respeta las agencias de todas las subjetividades, que pone en juego las dinámicas de poder que se establecen en esa relación. Se plantea como un proceso flexible, abierto a posibles modificaciones en curso, basada fundamentalmente en la reflexividad como estrategia. La IAF se instala en lógicas no propietarias del saber. Es decir, reconocemos el lugar de amplificadoras del conocimiento que nos posibilita la academia. Sin embargo, tenemos el desafío de encontrar otras formas de compartir el conocimiento producido más allá de las lógicas de autorías instaladas.

En nuestros procesos de investigación, también nos orienta la reflexividad, que como señala Rosana Guber (2001) es una herramienta fundamental en la medida que se convierte en una propuesta de reflexión-acción. Estrategia que también nos permite en palabras de Itziar Gandarias (2014b), la deconstrucción 
del poder, la co-creación del conocimiento y el abordaje de las dificultades y límites que nos encontramos en nuestras investigaciones.

Una reflexividad que se deja afectar y atravesar por las emociones como herramienta en la producción de conocimiento. Como señala Audre Lorde (1984:33):

"No es que la racionalidad no sea necesaria. Está al servicio del caos del conocimiento. Al servicio del sentimiento. Sirve para ir de un lugar a otro. Pero si no se concede valor a esos lugares, el camino no vale de nada. Y eso es lo que sucede muy a menudo con el culto a la racionalidad y con el pensamiento analítico, académico, circular. Aunque, en definitiva, yo no entiendo como una dicotomía el sentimiento y el pensamiento. Los entiendo como una elección de medios y combinaciones."

Nos referimos entonces a dejarnos tocar por la investigación, parafraseando a Mari luz Esteban (2004), transformando nuestra experiencia en palabras que compartimos en este artículo. Incorporamos por ello el análisis semióticomaterial de nuestros propios cuerpos en las investigaciones, como señala Daniela Osorio-Cabrera (2017), no para caer en la autorreferencia, sino para contemplar sus efectos en la producción del mismo.

Volviendo a la metáfora espiral, el objetivo de este artículo es realizar reflexiones teórico-epistemológicas colectivas en base a nuestras experiencias articuladas de investigación. Abordaremos tres dimensiones que se entrelazan en nuestros procesos de investigación y reflexión: lo político, lo ético y lo afectivo. Comprendemos estas dimensiones en su integralidad e interconexión, pero las señalamos de manera singular para rescatar algunos elementos particulares que han orientado nuestros itinerarios de investigación. Como última vuelta de la espiral, señalaremos la amistad como herramienta política en la producción de conocimiento.

\section{TRES DIMENSIONES PARA PENSAR UNA INVESTIGACIÓNN FEMINISTA: LO ÉTICO, LO POLÍTICO Y LO AFECTIVO.}

Las claves que mencionamos aquí, son parte de los intercambios construidos en este tiempo de trabajo juntas. Para nosotras en el inicio de nuestras investigaciones, la lectura de otras formas de hacer a la ciencia tradicional se volvió central para poder animarnos a investigar de otra manera. Por ello en este artículo las reflexiones se entretejen con pensadoras feministas, lo que otras investigadoras han dicho sobre investigación-activismo desde perspectivas feministas y lo que nosotras experimentamos. En definitiva, compartir, que de eso se trata cuando nosotras nos posicionamos desde los feminismos para pensar la producción de conocimiento. 


\subsection{Lo ético.}

Desde la filosofía feminista principalmente, se habla de la necesidad de pensar desde una nueva concepción y vivencia de poder y la emancipaciónn (Carosio 2007). Proponer una ética desde el feminismo implica una crítica al universalismo ético en sus formulaciones clásicas (Herrera 1992), un cuestionamiento radical a la idea de sujeto universal y al paradigma kantiano (Amorós 2001). La propuesta radica en la constitución de postulados que tomen en cuenta la realidad concreta, el contexto y la encarnación de cuerpos.

El debate en relación a una ética universal que sostiene los postulados de la ética tradicional, también se sostienen sobre la base de la dicotomíaa razónemoción (Carosio 2007). En este sentido, los planteos feministas apuestan por la radicalización de los afectos, como estrategia que supere la jerarquía de estas dicotomías. Una propuesta de estas características implica pensar en relación a las experiencias concretas. O como señala Diana Maffía (2005), la crítica éticofeminista se convierte en un instrumento que nos permite disminuir el androcentrismo y con él la opresión de género. La apuesta es recuperar al otro concreto, tomando distancia de formulaciones abstractas y del universalismo vacío, incorporando una lectura que lo aborde desde las diferencias y entramados de poder que se juegan en las relaciones (Herrera 1992).

En esta línea aparecen los planteos por una ética situada, una ética feminista que no se encarga solo de las mujeres, sino de aplicar algunos de los valores considerados "femeninos" y aportarlos para todos los seres humanos. En palabras de Alba Carosio (2007:168):

"Se trata de partir de la experiencia y modelar la experiencia desde la ética, en un juego entre realidad y trascendencia. En esta interacción surgirá la ética feminista como una ética del placer, placer de la realización y el despliegue humano".

En esta línea surgen debates como los planteados por Carol Gilligan (2013) en relación a la ética de la justicia y la ética de los cuidados. La ética de la justicia, como ética normativa, principio ordenador del sujeto universal, reglas universales que condicionan tanto el contrato social como los derechos naturales. La interpelación que hace el feminismo a la ética de la justicia, también se afirma en la crítica a la separación tradicional entre el espacio público y el privado, siendo el espacio público el de la justicia y buena vida, y el privado de los subtextos atravesados por el género.

La ética del cuidado viene a proponer este último como actitud y concepto, implica responsabilidad, valorar las relaciones personales y atender a la necesidad de otro/as. Con esta ética se incorpora la idea de pensar al otro/a como ser determinado, social-históricamente y la relación de proximidad y afectividad como comportamiento moral (Carosio, 2007). La propuesta no sería contraponer ambas éticas, sino la complementariedad que permita pensar una emancipación social que se concentren en la personalización y particularidad, evitando la des- 
personalización, la imparcialidad y la separación. Incluye incorporar la compasión en la idea de justicia.

Sin embargo, a esta propuesta no le han faltado críticas en tanto corre el riesgo de que se convierta en una vuelta a las condiciones de pérdida de autonomía para los cuerpos feminizados. Una de las voces que ha sonado más fuerte en este sentido es la de Celia Amorós (2007:79), "¡cuidado con la ética de los cuidados! Porque todos los boletos de la rifa están en el bombo que nos corresponde a nosotras". Considerando esta alerta, la autora (Amorós 2007) se apoya en la propuesta de Sartre de la ayuda en interrelación con la ética del cuidado feminista. Somos llamados a la ayuda de los otros en tanto somos libertades en situación. Por lo tanto no sería tanto un deber ser, sino un hacer. "Te pido ayuda en tanto mi libertad sin la tuya como la tuya sin la mía, no es nada" (Amorós 2007: 71).

Las premisas de una ética feminista situada y contextual, que cuestiona las dicotomías razón-emoción, público-privado, son las que han orientado las reflexiones de nuestros procesos de investigación y que desarrollamos a continuación. Una ética basada en las relaciones y los procesos son el eje orientador de nuestro trabajo, alertas a no reproducir la vuelta a una responsabilidad de los cuerpos feminizados en esta tarea. Destacamos dos dimensiones dentro de nuestra propuesta, una ética del acompañamiento que nos permita la constitución de circuitos de conocimiento feminista y la transparencia de nuestros procesos de investigación que den cuenta de las incomodidades como pistas para la producción de conocimiento.

\subsection{1. Ética del acompañamiento.}

Considerando estas discusiones filosóficas en torno a la ética feminista, promovemos una discusión de la ética en los procesos de investigación situados y relacionales. En nuestras investigaciones nos proponemos una ética del acompañamiento, tanto en relación con el acompañar los procesos colectivos, como entre nosotras como investigadoras. Queremos desplegar aquí algunas posibilidades y tensiones en este acompañamiento.

En relación al acompañamiento de los procesos colectivos en los que participamos, partimos de reconocer la agencia de las personas que participan en los procesos de investigación que desarrollamos. No buscamos la concientización ni la develación de hipótesis preelaboradas, sino que comprendemos nuestra participación como amplificadoras de procesos de reflexión (Biglia, 2005).

Este acompañamiento nos compromete a establecer un diálogo que permita procesos colaborativos en la producción de conocimiento. Sin embargo, no debemos perder de vista que estos procesos de producción conjunta, requieren de la escucha de los tiempos y ritmos colectivos. La tensión entre los tiemposexigencias de la academia y los del activismo se convirtieron en parte del recorrido. Por ejemplo mencionamos la tensión que se produce en la elaboración de textos colectivos que necesitan la revisión y aprobación del colectivo, cuando éste está saturado de trabajo. Elaborar estrategias flexibles que contemplen estos 
ritmos ha sido parte del aprendizaje. Nos referimos a no tensionar al colectivo para llegar a nuestras metas, reconocer cuándo es posible y cuándo no producir conjuntamente, identificar otras formas colaborativas y de participación que permitan la creación conjunta. Incluir en el análisis de los procesos de investigación estas tensiones, otra herramienta que incorporamos para trazar procesos de cocreación colectiva.

Nos referimos también en este proceso colaborativo de producción de conocimiento, a la constitución de circuitos de conocimiento feminista (Esteban 2014). Los circuitos que se establecen intentan romper la frontera academia activismo, aun a cuenta de ser consideradas demasiado activistas para la academia, o demasiado académicas para el activismo. Sin embargo, Esteban (2017) nos advierte sobre la influencia del fenómeno de "la cultura de las expertas" en el movimiento feminista, señalando la práctica de sacralizar a ciertas autoras feministas a las que se les asigna la autoría individual de conocimientos que en realidad han sido desarrollados de manera colectiva. Se establece así una jerarquía del conocimiento condicionada según el prestigio o glamour que tenga las autoras "expertas" dentro del movimiento feminista. Pareciera que si las investigaciones no se realizan bajo los parámetros y sello de la academia no tienen la misma validez ni el mismo reconocimiento, incluso también en el mundo de los movimientos sociales. Muchas veces nosotras fuimos convocadas como voceras de los colectivos en los que participamos-investigamos. Movernos del lugar de expertas, ceder espacios y generar procesos de autoformación dentro de los colectivos fueron algunas de las propuestas desplegadas para trabajar estas tensiones.

El otro acompañamiento refiere al que realizamos entre nosotras como investigadoras. Tomamos distancia de procesos individualizados y meritocráticos de investigación en la academia, para resaltar el lugar de la otra como punto de anclaje en nuestras reflexiones. Nuestro tránsito por el grupo "Fractalidades en Investigación Crítica" (FIC) de la UAB, constituyó un espacio catalizador de esta forma de intercambio cooperativo. Compartimos con otrxs compañerxs del doctorado e investigadoras nuestros trabajos, experiencias, reflexiones para construir pensamiento colectivo. Algunas de las reflexiones presentadas en este artículo, forman parte también del intercambio en otros espacios académicos sobre metodologías feministas como el SIMREF ${ }^{5}$ o la organización de actividades académicas sobre metodologías de investigación feminista. La propia constitución de "marietas" y la estrategia conjunta de trabajo e intercambio de saberes ha sido fundamental. El doctorado es un proceso que muchas veces se transita en solitario, poner en común nuestros aprendizajes, y en particular nuestros malestares ha compuesto este camino.

La epistemología de los conocimientos situados nos da una herramienta para la visibilización de nuestras formas de conocer. Como señala Haraway (1997:346), "la encarnación feminista, las esperanzas feministas de parcialidad,

${ }^{5}$ Seminario Interdisciplinar de Metodología de Investigación Feminista. http://www.simref. net/simref/ 
de objetividad y de conocimientos situados se vuelven conversación y códigos en este poderoso nudo en terrenos de cuerpos y significados posibles". Cuanta mayor diversidad establecemos en nuestras articulaciones, mayores posibilidades de la producción de un conocimiento que permita herramientas para la producción colectiva.

\subsubsection{La ética de la transparencia.}

La otra dimensión tiene que ver con romper la dicotomía público-privado, nos referimos a la transparencia de nuestros procesos de investigación. Hablamos de mostrar la "cocina" de la investigación feminista, lo que implica hacer transparentes las decisiones analíticas y metodológicas que tienen lugar en todo proceso de conocimiento.

Nuestras investigaciones se han basado en el paradigma cualitativo, y dentro de este, hemos abordado los procesos de investigación de forma flexible, en los que vamos tomando decisiones que condicionan dicho proceso. La herramienta de la reflexividad se destaca (Guber 2001) como principal analizador de las relaciones de poder y de cómo estas influyen en nuestras investigaciones visibilizando sus efectos.

Hablamos de las relaciones de poder que establecemos cuando nos articulamos con otras y los sentidos que nuestra posición adquiere en estas situaciones. En particular en el entorno activista somos interpeladas por el exceso de reflexión teórica, o la falta de experiencia práctica, sospechosas de apropiarnos de los conocimientos o de la mejora de nuestra nuestra condición de ascenso social gracias a los conocimientos que producimos (Osorio-Cabrera 2017). Contribuyen a la transparencia explicitar nuestras intenciones en relación al proceso y los cambios que se dan en el camino, compartir nuestros trabajos escritos, comprometernos en los procesos colectivos de producción.

La reflexividad también se nutre de nuestras incomodidades como herramientas para la producción de conocimiento (Gandarias 2014; Pilow 2003). Nos referimos a la toma de decisiones que desde nuestro cuerpo afectivo tomamos en los procesos de investigación. Dar cuenta de las decisiones prestando atención a aquellas situaciones de sentirse perdida, malestares, bloqueos, incongruencias como alertas para revisar cómo lo estamos haciendo (Gandarias 2014b).

Las interpelaciones a las que hacemos referencia forman parte del tránsito que realizamos como investigadoras y activistas, generando incomodidades tanto epistemológicas como corporales (Osorio-Cabrera 2017). Incomodidad en primer lugar epistemológica, pues la consideramos como parte de la interpelación en la producción de conocimiento. Nos referimos al desafío de producir conocimiento situado, y a la dificultad que se nos presenta muchas veces en no reproducir los modos tradicionales de hacer ciencia, estar alertas a no generar procesos "como sî".

Sobre las incomodidades corporales, nos dan pistas para orientarnos dentro de nuestros procesos de investigación. Sin embargo, nos hacen pasar malos mo- 
mentos, al interpelarnos en primera persona, pero funcionan como alerta, para reconocer cómo lo estamos haciendo. Nos referimos a cuándo es necesario parar y reelaborar, tomar decisiones que influyen de manera directa en nuestros recorridos. El proceso que construimos juntas nos permitió sostener estos momentos, generando redes de cuidado y autocuidado.

Atender a las incomodidades como señales que orientan las investigaciones y compartirlas nos permitió identificar momentos o situaciones que se convirtieron en herramientas para la producción conocimiento. Explicitar en el cuerpo de la tesis estos procesos se vuelve necesario, cuando generalmente quedan en la anécdota o a veces se incluyen en un apartado concreto de reflexión ética. La forma en la que transitamos las investigaciones está llena de estos momentos, visibilizarlos y compartirlos, nos brinda la oportunidad de construir de alguna manera a este testigo modesto mutado al que nos invita Haraway (1997).

Sin embargo, cuando decimos que es necesario visibilizar y reconocer estas incomodidades debemos mantenernos en alerta para no caer en procesos autorreferenciales o confesionales (Gandarias 2014b, Pilow 2003). El reconocimiento de los procesos que transitamos no tiene por qué convertirnos en las protagonistas de la historia. Con esto cobra más sentido la consigna feminista partir de sí, para no quedarse en sí (Precarias a la Deriva, 2004).

\subsection{Lo político.}

Además de la ética, las investigaciones feministas se caracterizan por su dimensión política donde las fronteras entre la producción de conocimiento y activismo se tornan difusas (Biglia 2006, Zavos y Biglia 2009). Para la academia feminista, la generación de conocimientos es un compromiso político en la construcción de alternativas de transformación radical. En consecuencia, como señala Patricia Castañeda (2019: 33) "hacer academia feminista es hacer política feminista".

Para ello, se torna indispensable mantener una vinculación constante entre teoría y praxis, donde el propósito central de ambas, es doble; por un lado, un continuo cuestionamiento del orden social dominante; desnaturalizándolo y mostrando las relaciones de poder que están detrás de su consolidación y por otro, una búsqueda incansable de alternativas a lo hegemónico (Balasch, Ema, y Gutiérrez 2005). En ese sentido, como investigadoras activistas feministas se hace fundamental reflexionar respecto a qué temas estudiamos, cuál es el rol de nuestras investigaciones, desde dónde nos planteamos la investigación y qué y cómo comunicamos.

Poner el foco en la vinculación entre teoría y praxis y, como consecuencia, en los efectos políticos de nuestras investigaciones conlleva inexorablemente una mayor responsabilidad (Banister, Burman, Parker, Taylor y Tindall 1994). Según Gayatri Spivak (1988) esa responsabilidad implica actuar desde la conciencia de los límites del propio conocimiento y de las posibilidades de acción. Tomando prestada la metáfora de Haraway (1995) como investigadoras nos hallamos en 
"la barriga del monstruo" de la academia, un lugar complejo en tanto que delimitado y conformado por las condiciones de posibilidad de los actuales contextos de producción de conocimiento, regidos por las lógicas competitivas y de mercado neoliberal que priorizan la eficacia constante y la hiperproductividad individual en detrimento de las relaciones de cooperación y reciprocidad (Amigot y Martinez 2013; Avila et al 2018).

No obstante, también ocupamos "una de las posiciones articulatorias desde las que se producen y generan significados y prácticas transformadoras social y subjetivamente" (Montenegro, Pujol y Vargas 2015: 1845). Habitar esos espacios desde la potencialidad de pensar, cuestionar y actuar sobre los límites mismos de las investigaciones genera nuevas formas de relación crítica dentro de la práctica investigadora que, en vez de evitar las controversias y las contradicciones, contribuye a visibilizarlas y a profundizar en su debate.

Hemos identificado dos elementos clave a la hora de pensar la dimensión política de las investigaciones activistas feministas: la articulación como estrategia para la transformación social y el continuum de transformar transformándonos.

\subsubsection{La articulación situada como estrategia para la transformación social.}

Ernesto Laclau y Chantal Mouffe (1987) definen la articulación como las relaciones semiótico-materiales entre entidades que se modifican y se constituyen en la misma relación. Es una perspectiva que cuestiona "el mito de que la realidad social está claramente definida y determinada" (Mumby y Putnam 1992:123) y aboga por la indeterminación, la incompletud y la inestabilidad como características de lo social.

Se trata de un concepto que entiende la actividad política de manera dinámica y aboga por la indecibilidad de lo social; esto es; el carácter contingente de los significados sociales (Montenegro, Rodriguez y Pujol 2014). La potencialidad y a la vez complejidad de la articulación radica en la apertura del tablero social ya que se trata de "una perspectiva que abre el juego político al reconocer que no hay un fundamento último de la acción a la vez que posibilita la acción política al definir fundamentos parciales, contingentes y temporales de la misma" (Montenegro y Pujol 2014:33).

Esta noción de articulación incide en el carácter relacional de las articulaciones. Esto hace imposible la fijación de las identidades ya que presupone una "presencia de unos objetos en otros" (Laclau y Mouffe 1987:118) lo que imposibilita el cierre de la identidad de los elementos articulados. Por lo tanto, la articulación cuestiona el sujeto político uniforme y monolítico y las grandes narrativas hegemónicas de la alternativa política, al mismo tiempo, que supone una negación de las dicotomías entre sujeto y objeto, investigadora/ participante (Dauder y Bachiller 2002). Estas dicotomías son oposiciones que reproducen 
las relaciones de poder a través de las prácticas y discursos dominantes de la investigación.

La figura de la articulación es estratégica para pensar nuestra posición dentro de las investigaciones como un nodo en una red más amplia que nos constituye y transforma, al mismo tiempo que contribuimos a su transformación (Montenegro, Pujol y Vargas 2015). Es una invitación a fomentar articulaciones donde participen posiciones de sujeto distintas, que pongan en cuestión las relaciones asimétricas que se reproducen en dichos procesos.

En ese sentido, pensar la construcción de conocimiento en términos de articulación es una apuesta política ya que como señalan Marisela Montenegro, Joan Pujol y Liliana Vargas (2015:1845):

"en la articulación se fraguan los límites de sujetos, opiniones, sentidos, valores y guías de acción; se definen procesos de inclusión y exclusión y se establecen conexiones (voluntarias e involuntarias) imbuidas en redes de poder, autoridad y definiciones previas que las delimitan, pero no las agotan".

Por tanto, la figura de la articulación situada nos permite una mayor amplitud e indeterminación a la hora de pensar los procesos de investigación, haciendo hincapié en las conexiones voluntarias e involuntarias de los agentes involucrados.

En nuestro caso, han sido varias las conexiones y articulaciones que hemos ido tejiendo. En el caso de Mujeres del Mundo y Sindillar, nuestras tesis han servido de puentes para que ambas organizaciones, una en Bilbao y otra en Barcelona se conozcan, compartamos experiencias y prácticas políticas y establezcamos un trabajo en red. Concretamente en junio del 2014, compañeras de Mujeres del Mundo viajamos a Barcelona para compartir con las compañeras de Sindillar un taller sobre vivencias, sentires y retos en el tejido de redes y alianzas. Meses más tarde, en noviembre del 2014, dos compañeras del sindicato Sindillar participaban en Bilbao en las Jornadas Somos muchas, diversas, enredadas y con propuestas para celebrar los 15 años de Mujeres del Mundo. Estos intercambios y encuentros además de compartir prácticas y conocimiento nos han permitido establecer alianzas para denunciar la falta de derechos de las mujeres migradas que trabajan en el trabajo del hogar y cuidados y tejer una red de apoyo mutuo entre Barcelona y Bilbao cuando llegan mujeres por primera vez a la ciudad o no cuentan con redes familiares.

En estos procesos de articulación cada agente, las investigadoras y las participantes, tienen un conocimiento parcial, situado, incompleto, imperfecto, momentáneo y único producido a través de la unión con un otro, pero sin pretender ser el otro (Haraway 1995).

Concebir la construcción de conocimiento desde esta perspectiva articulatoria y situada nos obliga a una "escucha desde la presencia", desde el aquí y el ahora; no tanto desde lo que buscamos escuchar para validar o refutar nuestras hipótesis o categorías teóricas previas, sino para estar abiertas a las diferencias y por ende a los conflictos inherentes que emergen a partir de ellas. Esto requiere desarrollar una política de múltiples reconocimientos articulatorios que fomente 
posibles escenarios para "hablar con la otra" y ser verdaderamente capaces de "escuchar a la otra". La necesidad, en términos de Spivak (1988:52), de una inteligibilidad mutua que "suspenda la creencia de que somos indispensables, mejores o culturalmente superiores; de resistir la tentación de proyectar nuestro mundo en el mundo del otro".

\subsubsection{Devenir activistas: transformar transformándonos.}

Nuestros procesos de investigación han estado marcados por un devenir activistas en las organizaciones donde hemos desarrollado nuestra tesis. A pesar de ser experiencias muy diversas, nos une que las tres hemos apostado por un compromiso radical con los contextos con los que nos hemos relacionado y esto ha tenido un impacto significativo en nuestras vidas personales que desbordó y continuó más allá de la realización de la tesis.

Algunas autoras denominan "poner el cuerpo" o "jugarse el cuerpo" a esta relación profunda en la que quien investiga no solo se involucra como estudiosa, sino también como activista, acompañante e integrante de las organizaciones con las que lleva a cabo sus indagaciones (Castañeda 2019, Fulchiron 2018; Gómez 2015).

Este jugarse el cuerpo ha significado una dificultad para movernos entre dos mundos que se relacionan y se miran escasamente: la academia y el activismo. Al igual que la poeta valeria flores ${ }^{6}$ (2014:5) en ocasiones también nos hemos sentido "demasiado intelectuales para el activismo, demasiado activistas para la academia". No queríamos que nos identificaran con la imagen tradicional de las investigadoras como usurpadoras del conocimiento. Con el propósito de evitar ser vistas como intrusas o "fuera de lugar" parafraseando a Nirmal Puwar (2004), nuestras prácticas han oscilado más hacia el lado del activismo; tratando de pasar desapercibidas hasta el punto de difuminarnos como investigadoras o involucrándonos de tal manera en las actividades de la organización que la realización de la tesis por un período de tiempo quedó pausada. Por ello, como señala Osorio-Cabrera (2017) en el equilibrio de posiciones, no podemos perder de vista nuestro rol de investigadora y trabajarla con el colectivo para que también esté presente.

En ese sentido, reivindicamos una posición híbrida y fronteriza alejándonos de las posiciones extremas y antagónicas que viven, o bien, a las espaldas de lo que acontece en el día a día de la realidad social o bien son reacias y rechazan cualquier estudio o investigación que venga del mundo académico. Apostamos por ser puentes entre el mundo académico y los movimientos sociales; traductoras del lenguaje académico enrevesado a un lenguaje más coloquial y cercano a la vida cotidiana. Es por ello que nos parece primordial generar espacios de encuentro y de construcción de conocimiento colectivo más allá del ámbito

${ }^{6}$ La autora escribe su nombre y apellido en minúsculas como una forma de resaltar la importancia de la obra o de lo que se dice, y no el nombre propio. 
universitario. Un ejemplo, fue la organización de la Jornada: ¿De qué hablamos cuando hablamos de metodologías feministas?, junto con el espacio La Bonne ${ }^{7}$ y el Sindicato Sindillar/Sindihogar que tuvo como objetivo compartir debates en torno al quehacer investigador desde metodologías y epistemologías feministas. Para ello, se organizó una mesa redonda con tres invitadas y un taller práctico ${ }^{8}$.

Movernos y habitar estos espacios fronterizos ha supuesto una acción política reflexionada y consciente del peligro actual de extractivismo epistémico (Grosfoguel, 2016) y academización de los espacios de investigación activista. De hecho, nos identificamos durante el proceso de nuestras tesis con la investigadora activista que describen (Araiza y González: 2017,68):

"no es equiparable ni al experto académico en movimientos sociales, ni al intelectual comprometido, ya que su cuerpo ha sido atravesado por un contexto de precarización, que se ha traducido en inestabilidad laboral, currículum no lineal y diverso, migración o exilio económico, entre otros".

Inestabilidad, incertidumbre y precariedad han marcado nuestros años de realización de la tesis. Las tres hemos pasado por diferentes situaciones; desde estar becadas para realizar el master y el primer año de doctorado, hasta compaginar trabajos precarios en fines de semana para poder seguir haciendo la tesis o buscar estrategias para seguir vinculadas al doctorado y poder así renovar el permiso de residencia para estudiantes extranjeros. Durante estos años el apoyo mutuo ha sido fundamental, de ahí que consideramos fundamental reflexionar sobre: “Quiénes hacen sostenibles nuestras investigaciones?”9

Tampoco queremos romantizar esta posición híbrida y presentarla como un salvavidas. Bajo una idealización de la investigación activista feminista (IAF) no podemos borrar las relaciones desiguales de poder que nos atraviesan. No deja de ser una posición incómoda que implica revisar los mecanismos que utilizamos para deshacernos del malestar de nuestro privilegio respecto a las participantes con posturas benevolentes y condescendientes hacia ellas, que acaban encubriendo relaciones de dominación.

Tomando la revolucionaria consigna "lo personal es político" de los movimientos feministas de los años 60 y 70, podemos concebir los procesos de investigación como una continuidad entre la investigadora y las personas participantes de la investigación (Gandarias, 2014a). Por ende, el campo de investigación es intensamente personal en el sentido de que no podemos esquivar las posiciones que ocupamos en base a los diferentes ejes de diferenciación. Además, la biografía de la investigadora y su relación con las participantes juega un papel central durante el transcurso de la investigación y también en la redacción final.

\footnotetext{
${ }^{7}$ La Bonne - Centre de Cultura de Dones Francesca Bonnemaison https://labonne.org.

${ }^{8}$ Puede accederse al video de la Jornada a través del siguiente enlace: https://www.youtube. com $/$ watch? $\mathrm{v}=4 \mathrm{~b} 99 \mathrm{KYrSgFg} \& \mathrm{t}=66 \mathrm{~s}$

${ }^{9}$ Con esta pregunta disparadora comenzamos nuestra presentación del Simposio: ¡Cuidado con los cuidados, pero sin mimos no hay revolución! que organizamos en el Congreso Internacional de Psicología Social Crítica: Afecto, Corporeidad y Política celebrado en la Universidad Autónoma de Barcelona del 12 al 14 de Febrero del 2015.
} 
Pero a su vez, si ampliamos lo político a la vida cotidiana, los procesos de investigación también son intensamente políticos, en términos de cómo las personas nos desenvolvemos en nuestro medio más cercano en el intento de cambiar las relaciones desiguales de poder. De esta manera, podemos afirmar que los procesos de investigación son viajes políticos personales y colectivos que transforman y nos transforman ya que como veremos a continuación no podemos habitar y afectar los lugares sin que los lugares nos habiten y afecten.

\subsection{Lo afectivo.}

Al hablar de afectos tenemos que situarnos en el largo recorrido que ha implicado el llamado "affective turn" o "giro afectivo". Si bien no es del interés de este artículo hacer una revisión exhaustiva de las diferentes tendencias del mismo, queremos mencionar su procedencia fundamental como parte de la investigación y de las emergentes metodologías dentro de las ciencias sociales (Ahmed 2015; Moraña y Sánchez Prado 2012).

En este sentido, la diversidad en la teoría de los afectos despliega una batería conceptual, que denota el interés que desde los años 80 , se ha manifestado por los aspectos emocionales (López, en Ahmed 2015). Mónica Greco y Paul Stenner (2008) explican este fenómeno desde diversos factores de acuerdo a la actual preeminencia de regímenes de sentimentalidad en ámbitos tan diferentes, como la justicia, los medios de comunicación, la política, los negocios, la educación o el sistema de salud.

Dentro del "giro afectivo" las discusiones, en parte, se han establecido en una diferenciación en relación a lo emocional y lo afectivo. La corriente que marca esta distinción como productiva, establece que las emociones conforman un sistema comunicativo integrado por medios expresivos, fisiológicos, conductuales y cognitivos construido culturalmente (Greco y Stenner 2008). Y por tanto, atravesados por múltiples intersecciones sociales como el género, la clase, la sexualidad, la raza, la situación migratoria, etc (López, en Ahmed 2015).

Por otro lado, el afecto, en la tradición spinozista, atiende a la pregunta: ¿cuánto puede un cuerpo? Se comprende que el modelo corporal no implica una desvalorización en ningún caso del pensamiento, sino que "cuando un cuerpo se encuentra con otro cuerpo distinto o una idea distinta, sucede que o bien ambas relaciones se componen generando algo más poderoso, o una de ellas descompone a la otra y destruye la cohesión entre sus partes" (Deleuze,1984:29).

En los estudios contemporáneos, esta autonomía semiótica del afecto, como lo explica Helena López (2015), es criticada por algunas autoras, entre ellas Sara Ahmed (2015), que plantean que la propia separación analítica entre emociones y afectividad puede implicar una reinstalación de la oposición, cultura/ naturaleza, que ignora el carácter sobredeterminado de los procesos corporales (Hemmings 2005).

En nuestras investigaciones, tomamos como eje dentro de la perspectiva de los afectos al ámbito de los cuidados. Especialmente consideramos la corriente 
de pensamiento de la economía feminista de la ruptura (Carrasco 2001; Pérez Orozco 2006) en su planteo de la sostenibilidad de la vida. Nos referimos a esta perspectiva para poder desarrollar una serie de marcos orientativos que nos ha aportado líneas de reflexión acerca de poner en el centro a las personas y el cuerpo en los procesos de investigación.

La sostenibilidad de la vida (Carrasco 2001) no solo nos plantea una forma de cubrir las necesidades materiales de existencia, sino que introduce aquellos elementos que permanecen ocultos como son los afectos y que son los que hacen posible que una vida valga la alegría de ser vivida. Este cambio de enfoque implica develar las falsas dicotomías entre lo productivo-reproductivo, privadopúblico, razón-emoción, deslizándose a posiciones que integran y amplían la noción de trabajo. Nosotras diríamos que podemos extrapolar a otros ámbitos de la vida, como la academia, donde también se hace imprescindible hacer visibles aquellas actividades históricamente excluidas como las tareas domésticas y de cuidados (Carrasco 2001).

Desde la economía feminista se ha puesto énfasis en recuperar la dimensión relacional y afectiva del estar-bien, considerando que las perspectivas más economicistas (Pérez Orozco 2014) obvian estas dimensiones. Descentrarse de la lógica del capitalismo contemporáneo y poner en el centro la vida, sin escencializarla. Es decir, no caer en un lugar utópico donde podría existir una vida allí fuera, fuera de las actuales relaciones de dominio o capital, sino preguntar(nos) bajo qué otros criterios y éticas se puede construir esa vida que merece la "alegría" vivir (Pérez Orozco 2012).

Así, nos propone una ruptura con la autosuficiencia heteropatrialcal, donde se mantiene bajo la alfombra la interdependencia de unas personas con otras a lo largo de nuestra existencia. Por lo tanto, lo que se plantea es un cambio ontológico donde se da la materialidad de la vida y los cuerpos, comprendiendo que la vida es vulnerable y finita (Pérez Orozco, 2006). Y de aquí se desprende que es necesaria una serie de circunstancias y relaciones que la hagan posible.

De esta forma poder retomar la comunidad o lo común, en tanto todas y todos somos seres que necesitan ser cuidados y cuidar para preservar la vida (Legarreta, 2012). Es decir, volver a colocar los cuidados en el centro, para intentar otro tipo de relaciones, que no estén supeditadas a las formas de dominación del capitalismo por desposesión, donde se valora la acumulación de propiedad intelectual, la concentración de conocimiento. Sobre todo un tipo de conocimiento, el del norte global, donde se moldea la forma de producción de conocimiento que es considerado útil para la reproducción del mismo.

En definitiva, es una invitación a estar atentas a las lógicas meritocráticas y de competitividad académica. Desenfocar la atención en lo utilitario del otro y la otra, como, por ejemplo, hacia las participantes de nuestras investigaciones; para ir hacia relaciones de cooperación y solidaridad mutua. Reconociendo que es un tipo de solidaridad asimétrica, en el sentido que no nos coloca a todas en una misma posición y no niega nuestras diferencias. Una solidaridad que no se confunde con paternalismos y formas de salvación de la otra, sino con la empatía 
y la afectación que provocan estar juntas ante las estructuras de múltiples desigualdades (género, raza, edad, sexo, clase, etc.)

Por ello, a continuación, haremos referencia a una serie de relaciones de cuidado y afecto que nos han atravesado en nuestros procesos de investigación, que son parte de nuestro conocimiento experiencial, con el deseo de compartirlos para que puedan acompañar otros caminos y formas de producir conocimientos.

\subsubsection{Relaciones de cuidado y autocuidado.}

Insiste Carol Gilligan (2013) en la necesidad de universalizar las obligaciones del cuidado, de hecho, puesto que somos mente y cuerpo, razón y emoción, la empatía de los humanos con sus congéneres debe darse por supuesta. No obstante, señala que tal suposición, queda dilatada ya que constata que la capacidad de empatía se pierde fácilmente.

En este sentido, los cuidados adquieren un valor en la conformación de las relaciones sociales y el lugar que ocupan en el orden social. La importancia de las relaciones y el cuidado, a diferencia de los estereotipos de género que suelen plantearse, no es un rasgo exclusivo de las mujeres, sino que también estaría presente en grupos étnicos minoritarios, colectivos en situación de exclusión, por mencionar algunas referencias (Tronto 1993, Puka 1993).

La propuesta es generar conocimiento desde otra racionalidad que involucre no sólo el pensamiento, sino las relaciones y el entramado de los afectos que estas producen. Dejarnos afectar, no dicotomizar los espacios, sino ponernos en relación, sumando y tensionando las diferencias. En resumen,

"se trata de enfatizar la importancia imperativa del vínculo afectivo, de comprender que las relaciones humanas son todas relaciones de [inter]dependencia, siempre frágiles y discontinuas. Estas relaciones son fundamentales para la adquisición de las competencias éticas y para convertirnos en seres humanos autónomos" (Alonso y Fombuena 2006:105).

No proponemos una visión romántica del cuidado, también reconocemos que el cuidado puede significar control, paternalismo en la relación con las personas con las que realizamos los procesos de investigación. Por ello es necesario desplegar una reflexividad que no tiña los procesos de nuestras inseguridades y reifique los roles de género y raza, construyéndonos en "salvadoras de situaciones", sino por el contrario, que nuestros cuerpos sean posibilidad de una re-construcción del vínculo afectivo-común. Que nuestros miedos, desánimo y nuestro no saber sea el puente para la construcción y el reconocimiento de nuestras diferencias y cuidados mutuos.

Cuando decimos autocuidado, nos referimos a hacer frente a las formas de producir conocimiento en las dinámicas capitalistas. Ante la idea de la virtud de la autosuficiencia, nosotras apostamos por colocar en el centro también nuestra vulnerabilidad y compartirla, como parte de una estrategia de construir en común (Silvia-Gil 2013). 
Invitamos a discutir las condiciones en las que investigamos, haciendo visibles las contradicciones que suceden en el frágil equilibrio entre los tiempos colectivos, los de la escritura y los de nuestra cotidianeidad. Es decir, qué tipo de materialidad está sosteniendo nuestros procesos de investigación, sin generar juicios de valor en torno a ello (becas, trabajo remunerado, paro), pero a sabiendas que las exclusiones de estos espacios también tienen que ver con ejes de desigualdad, en torno al sexismo, colonialismo y clasismo.

Por tanto, nos parece clave, generar procesos que sorteen las formas de creación individual, y experimentar procesos de creación colectiva y colaborativa, generando disidencias metodológicas a través de investigaciones posicionadas, horizontales y participativas, como formas de generar un tejido colectivo de autocuidados.

\subsubsection{Relaciones de confianza y de reciprocidad.}

Otras de las lógicas en las cuales nos vemos interpeladas en cuanto a las relaciones con otras, son aquellas que nos mantienen alertas ante lo diferente y/o extraño, y de lo cual hemos aprendido a desconfiar. La otredad se nos presenta como peligrosa de acuerdo a un tipo de socialización de la que hemos aprendido, en un mundo que trabaja constantemente hacia la hegemonía. De esta manera, los procesos de investigación son susceptibles de caer en estas lógicas, si no estamos atentas y en una revisión constante, acerca de no homogeneizar experiencias y de trabajar las diferencias. Esto contiene una doble arista, por un lado como oportunidades críticas entre investigadoras y activistas, y por otro lado, como una forma de nombrar las diferencias sociales, como construcciones de la otredad en relación al centro normativo.

Según nos plantea Rosi Braidotti (2004), en el principio no está la unidad, sino la alteridad, la estructura ontológica del sujeto se vincula inextricablemente con el otro, es decir, el sujeto se constituye no en su individualidad, sino en su interrelación con el afuera. Y, por tanto, nos vuelve a conectar con la interdependencia. Así, cuando nos involucramos con los colectivos y/o personas en nuestras investigaciones, podemos generar procesos de acompañamiento de nuestras extrañezas, a partir de lo diferente. Nos basamos en el respeto y honestidad mutua de conocernos y establecer relaciones de confianza, para hacer investigaciones críticas e interpelarnos desde estos lugares.

La confianza, sin embargo, no debemos entenderla como una instrumentalización para lograr unos objetivos individuales, sino que nos permite estar atentas a cuanto este vínculo sucede. Estar dispuestas a escuchar las críticas de nuestras formas de hacer y transformarlas, a cambiar el rumbo en base a los acuerdos que se generen. Conservar la intimidad del colectivo cuando este así lo disponga.

Estas relaciones, pueden ser propicias para generar pedagogías feministas en el proceso no solo de la investigación, sino que, como un efecto, de las mismas 
puedan estar sanando(nos), construyendo otro tipo de vínculos que potencien nuestra capacidad creadora.

El concepto de reciprocidad es un término que expresa el intercambio de bienes materiales y simbólicos y de trabajos en cualquier cultura, que, si bien ha sido utilizado por la antropología, se ha ampliado a las ciencias políticas y la economía (Esteban 2017). En el caso concreto de los procesos de investigación, nos parece pertinente que pueda ofrecernos claridad a la hora de hablar de los intercambios afectivos, que no necesariamente tienen que ser bidireccionales, sino que dicha reciprocidad pueda circular.

Nos referimos a relaciones que también se tornan extensivas cuando se construye una red de apoyos mutuos entre investigadoras, participantes, organizaciones y/o asociaciones, que acompañan y brindan solidaridad a su vez, estableciéndose una cadena de cuidados mutuos que es muy significativa (Juliano, 2014). Esto posibilita crear lazos de confianza, tejer alianzas y cooperar en la diversidad, unas con otras, con las dificultades que esto requiere, tanto políticas, raciales, económicas, entre los diferentes colectivos con los cuales nos involucramos como dentro de las propias organizaciones (Fulladosa 2017). En nuestro recorrido, lo hemos puesto en práctica, tomando decisiones políticas que implican ponernos en relación, con la posibilidad de que estas relaciones devinieran otras, y trascendieran nuestros intereses o propósitos como investigadoras.

Esta reciprocidad nos reorienta también en los compromisos asumidos, por eso también queremos resaltar que es importante la entrada al campo, o como nos gusta nombrarlo, desde la visión de que estamos abriendo un proceso en el desarrollo de la producción de conocimiento. Pero también es igual de importante, saber cerrar dichos procesos. En la investigación-activista nuestros cuerpos cobran ciertos significados en el espacio-tiempo, generan afectaciones singulares y colectivas que tenemos que tener presente a la hora de cerrar dicho proceso, y que va más allá del punto final de la tesis y de dichas investigaciones desde lo académico, tanto para las otras como para nosotras.

\section{A MODO DE CIERRE: LA AMISTAD COMO MODALIDAD PARA LA PRODUCCIÓN CONOCIMIENTO.}

Retomando la metáfora de la espiral, las tres dimensiones mencionadas se entretejen, por momentos superponen y desafían una lectura lineal en el propio texto. Las reflexiones teórico-epistemológicas sobre la ética, la política y los afectos resuenan con una idea central que atraviesa nuestros procesos y tiene que ver con la Amistad $^{10}$. Proponemos poner en el centro de este cierre la importancia de las redes de Amistad por lo que implica para nosotras en la producción de conocimiento. Nos apoyamos en la definición de Margarita Pisano quien

${ }^{10}$ Escribimos de manera consciente Amistad con la primera letra en mayúsculas para reforzar este tipo de lazos afectivos 
comprende la Amistad como un pensar juntas que se construye en los espacios fronterizos entre lo íntimo y lo público:

"la Amistad, se construye con un pie en lo privado y el corazón, y el otro, en lo público-político del pensar... del pensar juntas. Con todo lo que esta dimensión conlleva de valores y de responsabilidades sociales y humanas." (Margarita Pisano en Gaviola y Korol, 2015).

De la misma manera, Esteban (2017) enfatiza la importancia de estas redes de amistad para el sostenimiento de la vida; considera que son un compartir conjunto, no solo de cuidados, en el sentido habitual de este término, sino que traspasan las fronteras de las relaciones familiares actuales y acontecen en el "entre" y en el "a la vez" en diferentes espacios.

Entendemos la Amistad en la investigación como posibilidad para la creación de una ética feminista del acompañamiento, de transitar las dificultades y hacernos cómplices desde la intimidad y el goce. Acompañarnos también compartiendo los sinsabores de los procesos, las frustraciones, los conflictos y su impacto en todos los cuerpos involucrados. En definitiva, politizar nuestros malestares.

Una Amistad que toma la articulación situada como herramienta política para pensar nuestra posición dentro de las investigaciones en diálogo inacabado con múltiples posiciones de sujeto, que nos constituyen y transforman al mismo tiempo que contribuimos a la transformación social.

Una lógica de la Amistad que pretende romper con la lógica de la competitividad en la academia y el utilitarismo que se imponen en las relaciones en el contexto neoliberal. Una forma de compartir nuestras visiones que nos ayudan a ampliar los márgenes del conocimiento, donde poner a jugar la solidaridad, donde no llega una llega la otra. Construimos conocimiento desde la confianza que permita la crítica constructiva y la interpelación continua para repensar nuestros procesos.

Amistad que nos permita desarrollar investigaciones en clave de interdependencia, comprendiendo por una parte, la indisolubilidad de las dimensiones materiales y afectivas de las necesidades; por otro, la idea de cuestionar la dicotomía sostenimiento/necesidad; y finalmente, remarcar la importancia de los cuidados como necesidad básica de todas las personas a lo largo de todo el ciclo vital (Pérez Orozco 2012, Legarreta 2012). Cuidados entre todas las participantes de una investigación: de las investigadoras, los entornos con los cuales nos relacionamos, las directoras de tesis, las colegas y compañeras, las amigas y familias que nos acompañan, sin que esto genere una nueva moralidad.

En síntesis, y a modo de reflexión final, las reflexiones teórico-epistemológicas sobre la ética, la política y los afectos presentadas anteriormente nos interpelan a cuestionarnos cómo estamos construyendo el conocimiento y que ciencia merece la pena hacer y reivindicar. En ese sentido, apostamos por una radicalidad situada desde la "barriga del monstruo" de la academia y proponemos nuevas posibilidades de producción de conocimiento. Lo hacemos desde las experiencias corporales, los afectos y las diversas relaciones que vamos tejiendo 
y que sostienen nuestros procesos de investigación. Apostamos por construir colectivamente y en diálogo un conocimiento encarnado y situado, comprometido con la constitución de modos de vida vivibles.

\section{BIBLIOGRAFÍA.}

ADAN, CARME (2006): Feminismo y conocimiento, Coruña, Espiral Maior.

AHMED, SARA (2015): La política cultural de las emociones, México, PUEG-UNAM.

ALONSO ALONSO, ROSARIO y FOMBUENA V., JOSEFA. (2006): "La ética de la justicia y la ética de los cuidados", Portularia, (6), 1, pp. 95-107.

AMIGOT, PATRICIA Y MARTINEZ, LAUREANO. (2013): “Gubernamentalidad neoliberal, subjetividad y transformación de la universidad. La evaluación del profesorado como técnica de normalización". Athenea Digital 13(1): pp.99-120.

AMORÓS, CELIA (2001): Ética sarteana de la ayuda y ética feminista del cuidado: dos frentes críticos de la ética kantiana. En las Jornadas Internacionales "Dimensiones de la racionalidad práctica" Uned, Madrid, 5 diciembre 2001. Disponible en: www. uned.es/dpto fim/invfen/invFen4/celia.pdf

ARAIZA, ALEJANDRA Y GONZALEZ, ROBERT (2017): "La Investigación Activista Feminista. Un diálogo metodológico con los movimientos sociales", Empiria, Revista de metodología de ciencias sociales, (38), pp. 63-84.

ÁVILA, DEBORA, AYALA, ARIADNA \& GARCÍA, SERGIO. (2018). La Universidad y la vida., o cómo mantenernos vivos en medio de la neoliberalización de la Universidad. Disparidades. Revista De Antropología, 73(1), pp. 55-61. https://doi. org/10.3989/rdtp.2018.01.001.06

BALASCH, MARCEL, LÓPEZ, E. JOSÉ ENRIQUE, GUTIÉRREZ, M. PAMELA (2005): Globalización en la investigación en Psicología social y problemas sociales, Biblioteca Nueva, pp. 85-92.

BANISTER, PETER; BURMAN, ERICA; PARKER, IAN; TAYLOR, MAYE Y TINDAL, CAROL (1994): Métodos cualitativos en psicología, Guadalajara, Centro Universitario de Ciencias de la Salud.

BIGLIA, BARBARA (2005): Narrativas de mujeres sobre las relaciones de género en los movimientos sociales. Tesis Doctoral, Universidad de Barcelona, Barcelona.

BIGLIA, BARBARA (2006): “Some «Latin activist women' accounts»: Reflection on political research", Feminism \& Psychology, 16, (1), pp. 18-25.

BLAZQUEZ, NORMA (2011): “¿Cómo afectan las mujeres a la ciencia? El retorno de las brujas". En. (Blazquez, N) El retorno de las brujas. Incorporación, aportaciones y críticas de las mujeres a la ciencia, México, UNAM-CIICH, pp 97-130.

BRADIOTTI, ROSSI (2004): Feminismo, diferencia sexual y subjetividad nómade, Barcelona, Gedisa S.A.

CAROSIO, ALBA (2007): "La ética feminista. Más allá de la justicia”, Revista venezolana de estudios de la mujer, 12 (28), pp. 159-184.

CARRASCO, CRISTINA (2001): "La sostenibilidad de la vida humana: ¿un asunto de mujeres?", Mientras tanto, (82), pp. 3-22.

CASTAÑEDA, M. PATRICIA (2019): "Perspectivas y aportes de la investigación feminista a la emancipación" en Otras formas de desaprender: Investigación feminista en tiempos de violencia y resistencia, Bilbao, Hegoa, pp. 19-41.

DELEUZE, GILLES (2001): Spinoza: Filosofía práctica, Barcelona, Grafos S.A. 
ESTEBAN, MARI LUZ (2004). Antropología encarnada. Antropologi冈a desde una misma. Papeles del CEIC, 12, 1-21.

ESTEBAN, MARI LUZ (2014): "El feminismo vasco y los circuitos del conocimiento: el movimiento, la universidad y la casa de las mujeres". En Otras formas de (re)conocer: Reflexiones, herramientas y aplicaciones desde la investigación feminista, Bilbao: UPV/EHU, pp. 61-76.

ESTEBAN, MARI LUZ (2017): "Los cuidados, un concepto central en la teoría feminista: aportaciones, riesgos y diálogos con la antropología". Quaderns-e de l'Institut Català d'Antropologia, 22 (2),pp. 33-48.

FLORES, VALERIA (2014): Desmontar la lengua del mandato. Criar la lengua del desacato. Diálogo transfonterizo con tomás henríquez murgas y jorge díaz fuentes. Buenos Aires: Editorial Mantis.

FULCHIRON, AMANDINE (2018): La "ley de mujeres". Amor, poder propio y autoridad. Mujeres sobrevivientes de violación sexual en guerra reinventan la Justicia desde el cuerpo, la vida y la comunidad, Tesis doctoral en Estudios Latinoamericanos, UNAM, México.

FULLADOSA-LEAL, KARINA (2015): “Creando puentes entre la formación y la creatividad: Una experiencia de investigación activista feminista", Universitas Humanística, 79, pp. 115-140.

FULLADOSA-LEAL, KARINA (2017): Mujeres en movimiento: ampliando los márgenes de participación social y política en la acción colectiva como trabajadoras del hogar y el cuidado. Tesis doctoral en Psicología Social, UAB, Barcelona.

GANDARIAS GOIKOETXEA, ITZIAR. (2014a): Tensiones y distensiones en torno a las relaciones de poder en investigaciones feministas con Producciones Narrativas. Quaderns de psicologia 16 (1), 127-140.

GANDARIAS GOIKOETXEA, ITZIAR. (2014b): Habitar las incomodidades en investigaciones feministas y activistas desde una práctica reflexiva. Athenea Digital. Revista de pensamiento e investigación social, 14(4), pp. 289-304.

GARCÍA DAUDER, DAU y ROMERO BACHILLER, CARMEN. (2002): "Rompiendo viejos dualismos: De las (im)posibilidades de la articulación", Athenea Digital, 2, pp. 42-61.

GAVIOLA, EDDA, y KOROL, CLAUDIA (2015). A nuestras amigas. Sobre la amistad política entre mujeres. Chiapas: Prensa cartonera.

GILLIGAN, CAROL (2013): La ética del cuidado. Cuadernos de la Fundació Víctor Grífols i Lucas N 30. Barcelona, Fundació Víctor Grífols i Lucas.

GÓMEZ CORREAL, DIANA M. (2015): “De amor, vientre y sangre: Politización de lazos íntimos de pertenencia y cuidado en Colombia”, En Otras Palabras, 23, pp. 103-119.

GRECO, MONICA Y STENNER, PAUL eds. (2008): "Introduction: emotion and social science". En Emotions. A Social Science Reader, Londres, Routledge, pp. 1-21.

GROSFOGUEL, RAMÓN (2016): "Del extractivismo económico al extractivismo epistémico y ontológico", Revista Internacional de Comunicación y Desarrollo (RICD), 1(4), pp. 33-45.

GUBER, ROSANA (2001): La etnografía. Me冈todo, campo y reflexividad, Bogotá, Norma.

HARAWAY, DONNA (1995): Ciencia, Cyborgs y Mujeres. La Reinvención de la Naturaleza, Madrid, Cátedra.

HARAWAY, DONNA (1997): Testigo_Modesto@Segundo_Milenio. HombreHembra@_Conoce_Oncoratón®, Barcelona, UOC, 2004. 
HARDING, SANDRA (1996): Ciencia y Feminismo, Madrid, Morata.

HEMMINGS, CLARE (2005): "Invoking affect. Cultural theory and the ontological turn", Cultural Studies, 19, (5), pp. 548-567.

HERRERA LIMA, MARIA (1992): "La ética desde el feminismo. Notas sobre la diferencia”, Isegoría, 6, pp.153-160.

JULIANO, DOLORES (2014): Feminismo y sectores marginales: Un diálogo no siempre fácil [Comunicación Oral]. ERAPI, Laboratorio Cooperativo de Socioantropología, Barcelona.

KELLER, EVELIN (1991): Reflexiones sobre género y ciencia, Valencia, Edicions Alfons el Magnanim.

LACLAU, ERNESTO y MOUFFE, CHANTAL (1987): Hegemonía y Estrategia Socialista. Hacia una radicalización de la Democracia, Madrid, Siglo XXI.

LEGARRETA, MATXALEN (2012): El tiempo donado en el ámbito doméstico-familiar. Estudio sobre el trabajo doméstico y los cuidados, Tesis doctoral, Universidad del País Vasco.

LÓPEZ, G. de O. Helena (2015): Prólogo. en AHMED, Sara La política cultural de las emociones, México, PUEG-UNAM.

LÓPEZ-GIL, SILVIA (2013): Filosofía de la diferencia y teoría feminista contemporáneas: ¿cómo pensar la política hoy?, Tesis Doctoral, Departamento de Filosofía, Universidad Autónoma de Madrid.

LORDE, AUDRE (1984): La hermana, la extranjera. Artículos y conferencias, Madrid, Horas y horas.

MAFFIA, DIANA (2005): Éticas y Feminismos. Conferencia en el Instituto Interdisciplinario de Estudios de Género de la Facultad de Filosofía y Letras, Universidad de Buenos Aires, Buenos Aires, Argentina.

MONTENEGRO, MARISELA, RODRÍGUEZ, ALICIA, Y PUJOL, JOAN (2014): “La Psicología Social Comunitaria ante los cambios en la sociedad contemporánea: De la reificación de lo común a la articulación de las diferencias", Psicoperspectivas, 13(2), pp. 32-43.

MONTENEGRO, MARISELA, PUJOL, JOAN, Y VARGAS-MONROY, LILIANA (2015): "Miradas, formas de hacer y relaciones en la constitución de una investigación crítica”, Universitas Psychologica, 14(5), pp. 1833-1851.

MORAÑA, MABEL y SÁNCHEZ PRADO, IGNACIO eds. (2012): El lenguaje de las emociones. Afecto y Cultura en América Latina. Frankfurt: Iberoamericana / Vervuert.

MUMBY, DENNIS K., \& PUTNAM, LINDA (1992): "The politics of emotion: A feminist reading of bounded rationality", Academy of management review, 17(3), pp. $465-486$.

OSORIO-CABRERA DANIELA (2017): Modos de vida vivibles: Economia(s) Solidaria(s) y Sostenibilidad de la vida, Tesis doctoral en Psicología Social, UAB, Barcelona.

PÉREZ-OROZCO, AMAIA (2006): Perspectivas feministas en torno a la economía: el caso de los cuidados, Madrid: Consejo Económico y Social.

PÉREZ-OROZCO, AMAIA (2012): “De vidas vivibles y producción imposible”. En: No dejes el futuro en sus manos: Solidaridad internacional ante la crisis del capitalismo global, Barcelona: Entrepueblos, pp. 65-93. 
PÉREZ-OROZCO, AMAIA (2014): Subversión feminista de la economía, Madrid, Traficantes de Sueños.

PRECARIAS A LA DERIVA. (2004). A la deriva por los circuitos de la precariedad femenina, Madrid, Traficantes de Sueños.

PILLOW, WANDA (2003): "Confession, Catharsis, or Cure? Rethinking the Uses of Reflexivity as Methodological Power in Qualitative Research", International Journal of Qualitative Studies in Education, 16(2), pp. 175-96.

PUWAR, NIRMAL (2004): Space invaders: race, gender and bodies out of place, Oxford/New York, Berg.

PUKA, BILL (1993): “The liberation of Caring: A different voice for Gilligan's "Different Voice"'. En An ethic of care: feminist and interdisciplinary perspective London: Routledge, pp. 215-239.

SPIVAK, GAYATRI C. (1988): Can the subaltern speak? Reflections on the history of an idea, pp. 21-78.

TRONTO, JOAN C. (1993): "Beyond gender difference to a theory of care". En An ethic of care: feminist and interdisciplinary perspective, London: Routledge. pp. 240-257.

ZAVOS, ALEXANDRA y BIGLIA, BARBARA (2009): "Embodying feminist research: learning from action research, political practices, diffractions, and collective knowledge”, Qualitative research in psychology, 6, 1-2, pp.153-172. 